\title{
Power Limit for Crosswind Kite Systems
}

\author{
Mojtaba Kheiri ${ }^{1,2}$, Frédéric Bourgault ${ }^{1}$, Vahid Saberi Nasrabad ${ }^{1}$ \\ ${ }^{1}$ New Leaf Management Ltd., Vancouver, British Columbia, Canada \\ ${ }^{2}$ Concordia University, Department of Mechanical, Industrial and Aerospace Engineering, Montréal, Québec, Canada
}

\begin{abstract}
This paper generalizes the actuator disc theory to the application of crosswind kite power systems. For simplicity, it is assumed that the kite sweeps an annulus in the air, perpendicular to the wind direction (i.e. straight downwind configuration with tether parallel to the wind). It is further assumed that the wind flow has a uniform distribution. Expressions for power harvested by the kite is obtained, where the effect of the kite on slowing down the wind (i.e. the induction factor) is taken into account. It is shown that although the induction factor may be small for a crosswind kite (of the order of a few percentage points), neglecting it in calculations may result in noticeable overestimation of the amount of power harvestable by a crosswind kite system.
\end{abstract}

Keywords

Airborne wind energy, crosswind kite, induction factor, actuator disc, lift mode, drag mode, pumping kite, on-board generation

\section{INTRODUCTION}

Airborne Wind Energy (AWE) concerns accessing and harnessing high-altitude wind energy via either flying or aerostatic airborne devices such as balloons and kites usually tethered to the ground. Wind strength increases with altitude due to the decreasing effect of friction induced shear from the Earth surface. This is appealing because wind power density is proportional to wind velocity cubed (e.g. wind twice as strong $(2 \mathrm{X})$ means eight times $(8 \mathrm{X})$ the power). Winds at altitude are also more consistent, which helps to increase capacity factor. Various airborne concepts and principles have been proposed and exploited to reach these higher winds where electricity is typically generated either with on-board turbines or by pulling a load on the ground (e.g. unrolling the tether from a drum).

\section{Crosswind Kite Power Principle}

The principle of "crosswind kite power" was first introduced in a seminal paper by Miles Loyd (Loyd, 1980). A crosswind kite can harvest large amounts of wind power cheaply by means of an aerodynamically efficient tethered wing flying at high speed transverse to the wind direction in either lift mode (i.e. ground-based generation) or drag mode (i.e. on-board generation). It has been shown that the two harvesting modes are equivalent in terms of generating high power with little material. However, it is their practical implementation (and unique design challenges) which distinguishes them in terms of operation, performance, and cost of energy.

The fundamental concept of the crosswind principle is to exploit the glide ratio of the kite to induce a much higher apparent wind speed at the kite, unlike a static kite which is only subjected to the incoming wind. This phenomenon is sometimes referred to as aerodynamic gearing, which in turn increases the aerodynamic driving forces of the system by a square factor of the apparent wind. Both modes take advantage of this to extract power; increased thrust in drag mode versus increased pulling force in lift mode. Another advantageous effect of the crosswind principle is that it allows the kite to harvest power from a much larger capture area as it flies closed loop patterns compared to the static kite which only harvests from a region of the sky corresponding to its projected cross-section (and/or the rotor area of the turbine(s) it carries).

Simplistically, a crosswind system parallels a horizontal axis wind turbine (HAWT), where the kite traces a similar trajectory as the turbine blade tip (see Fig.1) ${ }^{1}$. For a HAWT, approximately half the power is generated by the last one third of the blade (Bazilevs, et al., 2011). To capture the same wind power, a kite does not require HAWT's massive hub and nacelle, steel tower and reinforced concrete foundation. The kite only requires a single lighter blade and lightweight tether(s) (e.g. made of ultra-high molecular weight polyethylene). Furthermore, reaching higher wind and/or increasing capture area for a HAWT incurs escalating costs from increasing tower and/or blade lengths; where the root loads upon these cantilevered beam elements are proportional to their length squared. In comparison, the increased tether cost for flying higher and/or widening the kite trajectory is insignificant. These characteristics combine to generate electricity with a Levelized Cost of Energy (LCOE) potentially near half that of conventional wind turbines, thus making wind power generation economically viable for a greater number of sites.

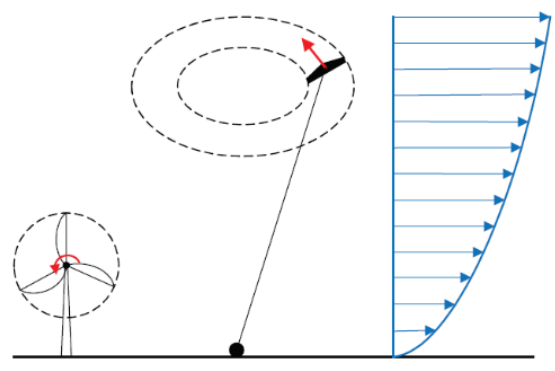

Figure 1. Capture area of a conventional HAWT (left) versus an AWE crosswind kite system (center). The arrows to the right illustrate typical wind velocity gradient. Half of the power generated by the HAWT comes from the last third of the blades but requires massive amounts of steel and concrete for support. The crosswind kite only needs a light tether, can cover a larger capture area and can reach more powerful winds at higher altitudes.

The pursuit of Crosswind Kite Power technology has only been made possible in recent time due to the advancements in electronics and control systems, light structural materials, tether technology, as well as the emergence of fully autonomous aerial vehicles. For a comprehensive review of airborne wind technologies and a list of some of the main players in the field from both academia and industry, the reader is referred to (Ahrens, Diehl, \& Schmehl, 2013); (Cherubini, 2015).

\section{Actuator Disc Theory and Betz-Joukowsky Power Limit}

It is well-known that there is a theoretical limit to the amount of harvestable power from a freestream via an energy extracting device. This limit is commonly referred to as the Betz-Joukowsky limit (Okulov $\&$ van Kuik, 2009) and may be derived from the actuator disc theory.

${ }^{1}$ Other types of trajectories such as figures-of-eight are possible. 
The actuator disc may be the first and simplest representation of a rotor, developed in the late 1800s and early 1900s by some prominent figures in fluid mechanics, such as Rankine, Froude, Joukowsky and Betz. According to the actuator disc theory, a rotor is represented by a permeable disc over which the load is distributed uniformly. Using the continuity and linear axial momentum equations, expressions for the axial force acting on the disc (i.e. thrust) and power harvested from flow are found. These expressions are essential for the preliminary design, performance prediction and load calculations of real rotors.

According to the actuator disc theory (Wilson \& Lissaman, 1974), the power coefficient, defined as the ratio of power extracted from flow to that available in an area equal to the disc's, is $C_{p}=4 a(1-a)^{2}$, where $a$ is called the induction or interference factor. The induction factor is a measure of the influence of the disc on the flow, and it may be correlated to the capability of the disc to harvest power from flow. In fact, the disc extracts power by slowing down the flow, and the induction factor serves as an indicator of flow deceleration. It follows from the above expression that the maximum value of $C_{p}$ is $16 / 27$, and it is achieved when $a=1 / 3$. In other words, power extraction from flow is maximized when the flow is decelerated in the vicinity of the disc to $1-a=2 / 3$ of the freestream. This shows that power extraction does not increase monotonically with the amount of flow deceleration, but rather reaches a limit before it starts decreasing.

In principle, a crosswind kite power system functions like a windmill, and it seems reasonable to use the actuator disc theory for performance prediction of the kite system. However, some researchers have expressed reservations about applying the Betz-Joukowsky limit to crosswind kite systems. For example, Loyd states that "the criteria for the efficiencies of a kite or its turbine are somewhat different from those used by Betz." He then neglects the induced effects of the kite slowing the wind, arguing that power is maximized when induction is minimized (i.e. for small kite over capture area ratio) and that the actuator disc efficiency of the kite is only a few percentage points; for more details, see (Loyd, 1980). Archer in Chapter 5 of (Ahrens, Diehl, \& Schmehl, 2013) confirms that the power coefficient of AWE systems is currently unknown, but she doubts the relevance of the BetzJoukowsky limit for AWE systems, claiming that the concept of a disclike swept area is not applicable. Also, (Costello, Costello, François, \& Bonvin, 2015) argue that the Betz-Joukowsky limit "cannot meaningfully be applied to kites" as the area swept by the kite is generally very large and the kite would only remove a small fraction of the available wind energy.

The objective of this paper is to apply the actuator disc theory to a crosswind kite system. For the sake of simplicity, a crosswind kite in straight downwind configuration with tether aligned with the wind is considered, where the kite sweeps an annulus perpendicular to the wind flow direction. It is also assumed that the kite is subjected to a uniform flow distribution. Expressions for the induction factor and harvested power in both lift and drag modes are obtained. Moreover, the importance of including the induction factor in crosswind kite systems calculations will be discussed.

\section{ACTUATOR DISC THEORY FOR A MOVING DISC}

Here, the actuator disc theory is extended to the case of a disc (i.e. rotor) moving at constant speed $v_{d}$, with respect to an inertial coordinate system, in the flow direction (see Fig.2). ${ }^{2}$ The motivation is

\footnotetext{
${ }^{2}$ The axial momentum theory has been applied to a wind turbine-driven vehicle by (Sorensen, 2016). The present derivation is, however, with greater elaboration.
}

to apply the extended theory to a crosswind kite system which, in general, may translate downwind while it is also moving crosswind (e.g. spiraling). This is characteristic of a crosswind kite system in lift mode during the power harvesting or reel-out phase of a pumping cycle.

Consider a control volume, CV1, enclosing an actuator disc of area $A$, which also coincides with the streamtube formed around the actuator disc. It is assumed that CV1 is moving with the disc at speed $v_{d}$. From the continuity equation, we may write

$$
\dot{m}=\rho A_{i}\left(v_{\infty}-v_{d}\right)=\rho A v_{r}=\rho A_{o}\left(v_{o}-v_{d}\right),
$$

where $\dot{m}$ represents the mass flow rate through the control volume, $v_{\infty}$ and $v_{o}$ are, respectively, the absolute flow velocities at sufficiently far upstream (i.e. inlet of CV1) and downstream (i.e. outlet of CV1) of the disc; $A_{i}$ and $A_{o}$ are, respectively, the flow area at the inlet and outlet of the control volume, and $v_{r}$ is the relative flow at the actuator disc.

By applying the linear momentum equation to CV1, we can obtain the axial thrust force, T, acting on the flow, the reaction of which is applied to the disc. That is

$$
-T=\rho A_{o} v_{o}\left(v_{o}-v_{d}\right)-\rho A_{i} v_{\infty}\left(v_{\infty}-v_{d}\right)=\dot{m}\left(v_{o}-v_{\infty}\right),
$$

in which it has been assumed that the control volume is under a uniform external pressure $p_{\infty}$, and thus zero net pressure force acting on the boundaries of the control volume.

Furthermore, the linear momentum equation may be applied to the control volume, CV2, enclosing only the disc:

$$
T=\left(p_{d-}-p_{d+}\right) A,
$$

where it has been assumed that the flow velocity does not change across the actuator disc, only the pressure does; $p_{d-}$ and $p_{d+}$ represent, respectively, the static pressure just before and after the disc.

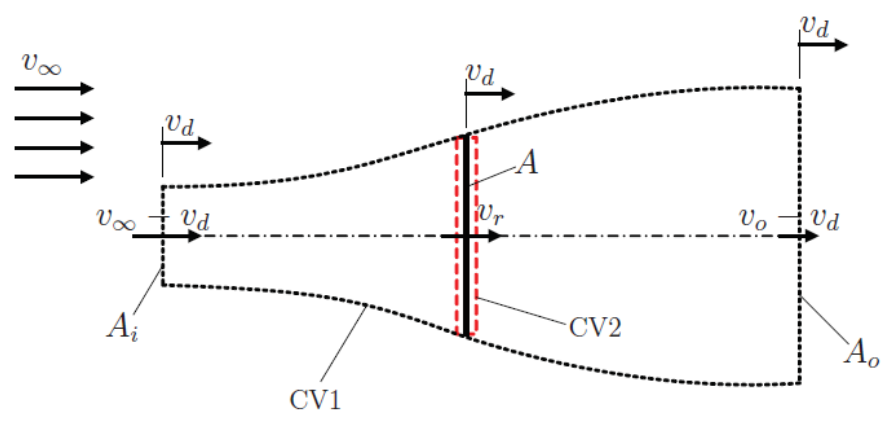

Figure 2. A schematic showing an actuator disc (solid line) of area $A$ exposed to wind flow velocity $v_{\infty}$, (from the left) and moving downwind at constant speed $v_{d} . \mathrm{CV} 1$ (dotted black line) represents the control volume enclosing the actuator disc, which also coincides with the streamtube, while CV2 (dashed red line) is the control volume enclosing only the disc; both control volumes move with the disc at constant speed $v_{d} ;\left(v_{\infty}-v_{d}\right)$ is the velocity of flow entering CV1 through inlet cross-section area $A_{i} ; v_{r}$ is the (relative) flow velocity at the actuator disc, and $\left(v_{o}-v_{d}\right)$ is the velocity of flow leaving CV1 through outlet area $A_{o}$.

Consider a streamline extending from the inlet to the actuator disc and another streamline from the disc to the outlet, and apply Bernoulli's equation to these streamlines. As shown in the Appendix, the Bernoulli equations for these streamlines may be written as: 


$$
\begin{aligned}
& p_{\infty}+\frac{1}{2} \rho\left(v_{\infty}-v_{d}\right)^{2}=p_{d-}+\frac{1}{2} \rho v_{r}{ }^{2} \\
& p_{d+}+\frac{1}{2} \rho v_{r}{ }^{2}=p_{o}+\frac{1}{2} \rho\left(v_{o}-v_{d}\right)^{2}
\end{aligned}
$$

which show that relative flow velocities appear in the dynamic pressure terms

Using Eqs. 4 and 5 and the fact that $p_{o}=p_{\infty}$ (according to the actuator disc theory, it is assumed that the outlet is sufficiently far from the disc such that the static pressure is recovered and reaches $p_{\infty}$ ), we can obtain $\left(p_{d_{-}}-p_{d_{+}}\right)$as

$$
p_{d-}-p_{d+}=\frac{1}{2} \rho\left[\left(v_{\infty}-v_{d}\right)^{2}-\left(v_{o}-v_{d}\right)^{2}\right] .
$$

Substituting Eq.6 into Eq. 3 yields

$$
T=\frac{1}{2} \rho A\left[\left(v_{\infty}-v_{d}\right)^{2}-\left(v_{o}-v_{d}\right)^{2}\right]
$$

By equating Eqs. 2 and 7 and letting $\dot{m}=\rho A v_{r}$ (refer to Eq. 1), one may obtain

$$
v_{r}=\frac{1}{2}\left[\left(v_{\infty}-v_{d}\right)+\left(v_{o}-v_{d}\right)\right]
$$

which means that the relative flow velocity at the actuator disc is the average of the inlet and outlet relative flows.

It appears reasonable to define the induction or interference factor $a$ in connection to the relative flow velocity, as

$$
v_{r} \equiv\left(v_{\infty}-v_{d}\right)(1-a)
$$

Eq. 9 means that retardation/deceleration happens to the relative flow/wind $\left(v_{\infty}-v_{d}\right)$. Using Eqs. 8 and 9 and letting $v_{d}=e v_{\infty}$, one may obtain

$$
v_{o}=v_{\infty}[1-2 a(1-e)]
$$

Thus, the axial force or thrust (Eq. 7) may be re-written as

$$
T=\left(\frac{1}{2} \rho A v_{\infty}^{2}\right) 4 a(1-a)(1-e)^{2} .
$$

Eq. 11 shows that the thrust of a moving actuator disc is the same as a stationary actuator disc scaled by a factor $(1-e)^{2}$. In other words, to find the thrust of an actuator disc moving with the constant speed of $v_{d}=e v_{\infty}$, one may simply replace $v_{\infty}$ in the thrust formula for a stationary disc by $v_{\infty}-v_{d}=(1-e) v_{\infty}$, i.e. the relative freestream velocity.

The power harvested by the actuator disc may be obtained as

$$
P=T v_{r}=\left(\frac{1}{2} \rho A v_{\infty}^{3}\right) 4 a(1-a)^{2}(1-e)^{3} .
$$

Similarly, one may conclude that the power harvested by an actuator disc moving at constant speed $v_{d}=e v_{\infty}$ may be obtained from the power formula for a stationary disc, provided that $v_{\infty}$ is replaced by $(1-e) v_{\infty}$.

The thrust coefficient $C_{T}$ and the power coefficient $C_{P}$ for a moving disc/rotor may be defined as follows

$$
\begin{gathered}
C_{T}=\frac{\left(\frac{1}{2} \rho A v_{\infty}^{2}\right) 4 a(1-a)(1-e)^{2}}{\frac{1}{2} \rho A v_{\infty}^{2}}=4 a(1-a)(1-e)^{2}, \\
C_{P}=\frac{\left(\frac{1}{2} \rho A v_{\infty}^{3}\right) 4 a(1-a)^{2}(1-e)^{3}}{\frac{1}{2} \rho A v_{\infty}^{3}}=4 a(1-a)^{2}(1-e)^{3} .
\end{gathered}
$$

Finally, the efficiency of the actuator disc may be defined as the ratio of extractable power to the wind power available to the disc (refer to (Wilson \& Lissaman, 1974)):

$$
\eta_{A D}=\frac{\left(\frac{1}{2} \rho A v_{\infty}^{3}\right) 4 a(1-a)^{2}(1-e)^{3}}{\left(\frac{1}{2} \rho A v_{\infty}^{3}\right)(1-a)(1-e)^{3}}=4 a(1-a) .
$$

\section{INDUCTION FACTOR FOR A CROSSWIND KITE}

The annulus flow derivation presented here follows similar steps as (Wilson \& Lissaman, 1974). For simplicity, it is assumed that the induction factor in the lateral/crosswind direction is negligible (i.e.á $=$ 0 ). Normally, the axial induction factor $a$ is found at any radial distance $r$ from the center of rotation. This is performed by equating the axial force generated in the annular element of width $\mathrm{d} r$ to the axial force predicted by the blade element aerodynamic considerations. Here, instead of finding local axial induction factors, an average induction factor is obtained, assuming that the velocity of the kite in the lateral (crosswind) direction is constant along its span. ${ }^{3}$

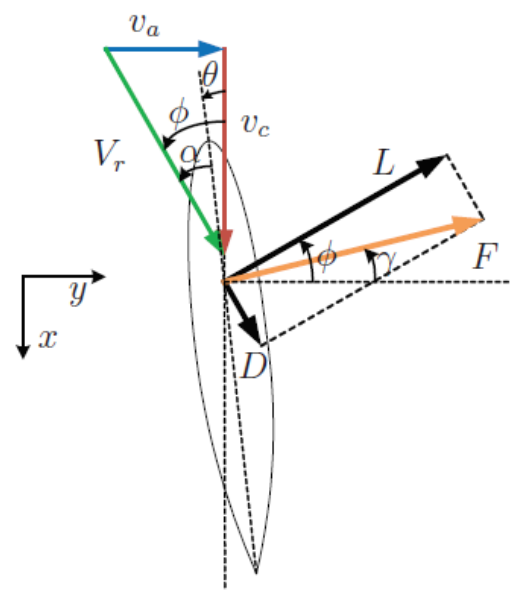

Figure 3. Velocity vectors and aerodynamic forces on a section of a crosswind kite: $v_{a}, v_{c}$, and $V_{r}$ represent, respectively, the axial, lateral, and total relative fluid-kite velocities; $L, D$, and $F$ are, respectively, the lift, drag, and total aerodynamic forces; $\alpha$ is the angle of attack, $\theta$ is the pitch angle, $\gamma$ is the angle between $F$ and the $y$-direction, and $\varnothing=\theta+\alpha$ is the angle between $V_{r}$ and the $x$-direction.

Fig. 3 shows a section of the kite with the aerodynamic forces acting on it, where $L$ is for lift, $D$ for drag and $F$ is the resultant force. For simplicity, we consider a straight downwind configuration, where the kite sweeps an area perpendicular to the wind direction. As shown in the figure, $V_{r}$ is the relative flow velocity in the vicinity of the airfoil section, and $v_{a}$ and $v_{c}$ are, respectively, the axial and lateral/crosswind components of $V_{r}$. Also, $\theta$ is the pitch (or twist) angle, $\alpha$ is the angle of attack, $\gamma$ is the angle between $F$ and the $y$-direction, and $\varnothing=\theta+\alpha$ is the relative wind angle with the crosswind plane.

${ }^{3}$ In fact, further assumptions are required to obtain the average axial induction factor; for instance, the kite has a rectangular planform, and the airfoil sections along the span are of the same shape. 
Equating the axial force generated in the annular area, $A_{s}$, swept by the kite (refer to Eq. 11) to the total axial aerodynamic force acting on the kite yields

$\frac{1}{2} \rho A_{k} V_{r}^{2}\left(c_{L} \cos \emptyset+c_{D} \sin \emptyset\right)=\frac{1}{2} \rho A_{s} v_{\infty}^{2} 4 a(1-a)(1-e)^{2}$,

where $A_{k}$ is the kite planform area; $c_{L}$ and $c_{D}$ are the lift and drag coefficients of the kite. ${ }^{4}$

Using Fig. 3, one may obtain $\gamma$ as

$$
\gamma=\tan ^{-1}\left(\frac{v_{a}}{v_{c}}\right)-\tan ^{-1}\left(\frac{c_{D}}{c_{L}}\right)
$$

which confirms that $\gamma \cong 0$ when $\left(v_{a} / v_{c}\right),\left(c_{D} / c_{L}\right) \ll 1$. Assuming that this condition holds, one may write

$$
\tan \emptyset=\frac{v_{a}}{v_{c}} \cong \frac{c_{D}}{c_{L}}
$$

or alternatively,

$$
v_{c}=\frac{c_{L}}{c_{D}}(1-a)(1-e) v_{\infty}
$$

in which $v_{a}$ has been replaced with $v_{\infty}(1-a)(1-e)$.

Eq. 16 may be re-written as

$\frac{1}{2} \rho A_{k}\left(v_{a}^{2}+v_{c}^{2}\right)\left(c_{L}+c_{D} \frac{c_{D}}{c_{L}}\right) \cong \frac{1}{2} \rho A_{s} v_{\infty}^{2} 4 a(1-a)(1-e)^{2}$,

in which $\cos \emptyset \cong 1$ and $\sin \emptyset \cong \tan \emptyset \cong\left(c_{D} / c_{L}\right)$ have been utilized.

The left-hand side of Eq. 20 may be further simplified, assuming that $\left(v_{a} / v_{c}\right) \ll 1$ and the fact that $c_{D}\left(c_{D} / c_{L}\right) \ll c_{L}$, as

$$
\frac{1}{2} \rho A_{k} v_{c}^{2} c_{L} \cong \frac{1}{2} \rho A_{s} v_{\infty}^{2} 4 a(1-a)(1-e)^{2},
$$

By substituting Eq. 19 into Eq. 21, the following equation yields

$$
\frac{a}{1-a} \cong \frac{1}{4}\left(\frac{A_{k}}{A_{S}}\right) c_{L}\left(\frac{c_{L}}{c_{D}}\right)^{2}
$$

Eq. 22 gives the average axial induction factor for a kite with a planform area of $A_{k}$, swept area of $A_{s}$ and aerodynamic coefficients $c_{L}$ and $c_{D}$. The area ratio $A_{k} / A_{s}$ may, in fact, be called the solidity factor of the kite, in accordance with terminology used for wind turbines. It can easily be concluded from Eq. 22 that increasing either the solidity factor, $c_{L}$, and/or $c_{L} / c_{D}$ will increase the induction factor.

\section{LIFT MODE POWER}

Here, starting from the expression found for the axial force or thrust acting on the moving actuator disc (Eq. 11), one may find the expression for the harvestable wind power from a kite in lift mode (during the harvesting phase of a pumping cycle ${ }^{5}$ ). The expression for harvested power is

\footnotetext{
${ }^{4}$ In the present formulation, in general, $c_{D}$ also includes the drag coefficient of the tether, in addition to the drag coefficient of the kite. ${ }^{5}$ To obtain the net average kite power over a pumping cycle, $P_{C}$, one has to also take into account the power required for the retraction or reel-in phase, $P_{r i}$, as well as the time required for each phase, in a weighted sum as follows: $P_{C}=\frac{P_{L} t_{L}+P_{r i} t_{r i}}{t_{L}+t_{r i}}$ where $t_{L}$ and $t_{r i}$ are the reelout and reel-in period, respectively.
}

$$
P_{L}=T v_{d}=\left(\frac{1}{2} \rho A_{s} v_{\infty}^{3}\right) 4 a(1-a)(1-e)^{2} e,
$$

in which it is recalled that $A_{s}$ is the area swept by the kite.

Using Eqs. 22 and 23, the lift power may be expressed in terms of kite's planform area and its aerodynamic characteristics:

$$
\begin{gathered}
P_{L}= \\
\left(\frac{1}{2} \rho A_{s} v_{\infty}^{3}\right) 4\left(\frac{1}{4}\left(\frac{A_{k}}{A_{s}}\right) c_{L}\left(\frac{c_{L}}{c_{D}}\right)^{2}(1-a)\right)(1-a)(1-e)^{2} e \\
=\left(\frac{1}{2} \rho A_{k} v_{\infty}^{3}\right) c_{L}\left(\frac{c_{L}}{c_{D}}\right)^{2}(1-a)^{2}(1-e)^{2} e .
\end{gathered}
$$

Letting $a=0$ in Eq. 24 results in Loyd's expression for the lift mode power. If we assume that $a$ and $e$ are independent, it follows that the maximum $P_{L}$ is achieved when $e=1 / 3$. Thus, the maximum lift mode power may be obtained as

$$
P_{L, \text { max }}=\frac{4}{27}\left(\frac{1}{2} \rho A_{k} v_{\infty}{ }^{3}\right) c_{L}\left(\frac{c_{L}}{c_{D}}\right)^{2}(1-a)^{2} .
$$

Eq. 25 shows that neglecting a small induction factor of a few percent in power calculations may result into a significant overestimation of the maximum amount of harvestable power in lift mode. For example, neglecting a $5 \%$ induction factor results in nearly $10 \%$ overestimation.

\section{DRAG MODE POWER}

In drag mode, power extraction does not happen by pulling a load downwind; rather, power is produced by loading the kite with additional drag: on-board turbines carried by the kite use the high relative wind speed to generate power. In this section, the extended form of actuator disc theory is used to obtain the expression for power extracted by the kite in drag mode (i.e. with on-board generation).

It is assumed that the on-board turbines have a total thrust force, $T_{t}$, which is a factor $\kappa$ times the drag of the kite (i.e. $T_{t}=\kappa D$ ). The power harvested by the turbines then may be written as

$$
P_{D}=T_{t} v_{c}=\kappa D v_{c} \cong \frac{1}{2} \rho A_{k} v_{c}^{3} \kappa c_{D}
$$

where $V_{r} \cong v_{c}$ has been used.

Eqs. 19 and 22 should be re-written for a kite in drag mode (note that $e=0$ in this case); they are

$$
\begin{gathered}
v_{c}=\left(\frac{c_{L}}{c_{D}+\kappa c_{D}}\right)(1-a) v_{\infty}=\left(\frac{1}{1+\kappa}\right) \frac{c_{L}}{c_{D}}(1-a) v_{\infty}, \\
\frac{a}{1-a} \cong \frac{1}{4}\left(\frac{A_{k}}{A_{S}}\right) c_{L}\left(\frac{c_{L}}{c_{D}+\kappa c_{D}}\right)^{2}=\frac{1}{4}\left(\frac{A_{k}}{A_{S}}\right) c_{L}\left(\frac{c_{L}}{c_{D}}\right)^{2}\left(\frac{1}{1+\kappa}\right)^{2} .
\end{gathered}
$$

Eq. 28 shows that the induction factor for the kite in drag mode is a function of $\kappa$, in addition to also being a function of the solidity factor and the lift and drag coefficients (cf. Eq. 22). Substituting Eq. 27 into Eq. 26 yields the following extended power equation for drag mode:

$$
P_{D}=\left(\frac{1}{2} \rho A_{k} v_{\infty}^{3}\right) c_{L}\left(\frac{c_{L}}{c_{D}}\right)^{2}(1-a)^{3} \frac{\kappa}{(1+\kappa)^{3}} .
$$

Letting $a=0$ in Eq. 29 results in Loyd's expression for the harvested power in drag mode. For a negligible induction factor, it follows that the maximum $P_{D}$ is achieved when $\kappa=1 / 2$. The expression for the 
maximum drag mode power for a non-negligible induction factor, in contrast to lift mode, becomes complex, and this is because the induction factor is also a function of $\kappa$ (refer to Eq. 28). For the sake of brevity, that expression is not given here.

The drag mode power may be expressed as a function of the swept area using Eqs. 28 and 29; that is

$$
P_{D}=\left(\frac{1}{2} \rho A_{s} v_{\infty}^{3}\right) 4 a(1-a)^{2} \frac{\kappa}{1+\kappa} .
$$

\section{NUMERICAL RESULTS}

In this section, some selected numerical results are presented for crosswind kites in both lift and drag modes. More specifically, variation of the normalized power (i.e. $\left.P /\left(1 / 2 \rho A_{k} v_{\infty}{ }^{3}\right)\right)$, power overestimation (i.e. $\left(P_{\text {Loyd }}-P\right) / P_{\text {Loyd }} \times 100$, where $P_{\text {Loyd }}$ is the power predicted by Loyd's expressions), and the induction factor are given as a function of (a) the ratio of reel-out speed to freestream velocity for a lift mode kite, and (b) the ratio of on-board turbines thrust to kite drag for a drag mode kite.

\section{Crosswind Kite in Lift Mode}

Fig. 4 shows variation of the normalized power in lift mode as a function of the ratio of reel-out speed to freestream velocity, $e$, for different values of the solidity factor typical of crosswind kite systems (i.e. $\sigma=A_{k} / A_{s}=0.001$ to 0.01 ). It is assumed that $c_{L}=1.0$ and $\left(c_{L} / c_{D}\right)=10$ (note that $c_{D}$ also includes the tether drag) for all the curves in this figure and in all subsequent figures. For comparison purposes, the normalized power curves for $\sigma=0$ and $\sigma=0.0345$ were also shown in the figure. A solidity factor of zero corresponds to Loyd's formulation, ${ }^{6}$ and $\sigma=0.0345$ is the solidity factor for a typical, modern, three-blade conventional wind turbine (Burton, Sharpe, Jenkins, \& Bossanyi, 2001).

As seen from Fig. 4, by increasing the solidity factor (meaning decreasing the size of the annulus swept by the kite), the normalized power decreases. This is to be expected as a higher solidity factor means a smaller swept area (thus less available power) for the same kite area. The peak power is decreased by $33 \%$ when the solidity factor is increased from 0.001 to 0.01 . Also, the peak power occurs at $e=1 / 3$, and it is independent of the solidity factor. Notice how much less power is generated as the solidity of the crosswind kite approaches that of a conventional wind turbine.

Fig. 5 shows how much overestimation is made in lift power calculation if one uses Loyd's formulation (i.e. neglecting the induction factor) instead of the formulation presented in this paper. For example, a realistic solidity factor for a $2 \mathrm{MW}$ pumping kite system may be approximately 0.005 , and the corresponding power overestimation for such kite would be around 21 percent. As seen from the figure, the overestimation is strongly dependent on the solidity factor: it becomes more significant when the solidity factor is increased. As also seen, the amount of overestimation is independent of the ratio of reel-out speed to freestream velocity.

Fig. 6 shows variation of the induction factor in lift mode, $a_{L}$, as a function of the ratio of reel-out speed to freestream velocity, $e$, for different values of the solidity factor, $\sigma$. As seen from the figure, except for very small values of $\sigma$, the induction factor is not negligible. For example, for $\sigma=0.005$, the induction factor is about 0.11 . As also seen, the induction factor in lift mode is independent of $e$, but it increases as the solidity factor is increased. An induction factor of zero corresponds to Loyd's result.

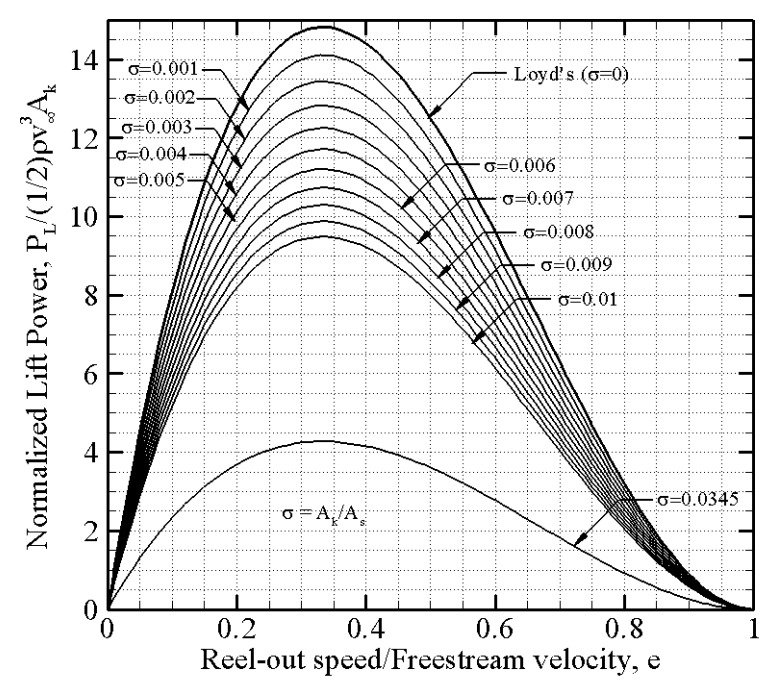

Figure 4. Normalized lift power as a function of the ratio of reel-out speed to freestream velocity, $e$, for different values of the solidity factor, $\sigma$. For all the curves, $c_{L}=1.0$ and $\left(c_{L} / c_{D}\right)=10$.

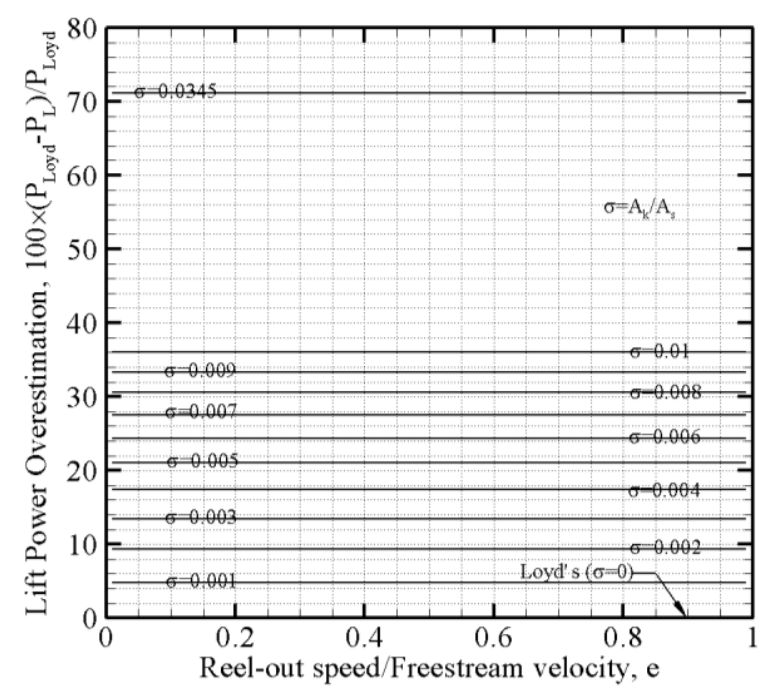

Figure 5. Lift Power overestimation as a function of the ratio of reel-out speed to freestream velocity, $e$, for different values of the solidity factor, $\sigma$. For all the curves, $c_{L}=1.0$ and $\left(c_{L} / c_{D}\right)=10$. 


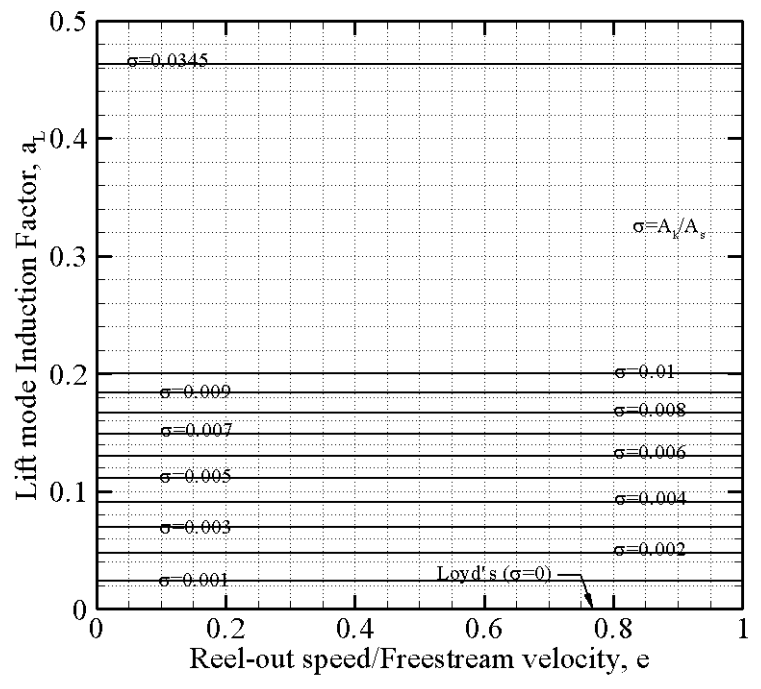

Figure 6. Induction factor in lift mode as a function of the ratio of reel-out speed to freestream velocity, $e$, for different values of the solidity factor, $\sigma$. For all the curves, $c_{L}=1.0$ and $\left(c_{L} / c_{D}\right)=10$.

\section{Crosswind Kite in Drag Mode}

Fig. 7 shows variation of the normalized power in drag mode as a function of the ratio of thrust of on-board turbines to kite drag, $k$, for a typical range of the solidity factors, i.e. $\sigma=A_{k} / A_{s}=0.001$ to 0.01 . Here again, curves for $\sigma=0$ (Loyd's formulation) and $\sigma=0.0345$ (modern HAWT) are shown for comparison purposes.

As seen from the figure, similarly to the lift mode power, the normalized power in drag mode decreases as the solidity factor is increased. The peak power is decreased by approximately $22 \%$ when the solidity factor is increased from 0.001 to 0.01 . In contrast to the lift mode power, the peak power occurs at a different $\kappa$ as the solidity factor is varied. This is because the induction factor for a drag mode kite is also dependent on $\kappa$, as also previously explained. As seen from the figure, the peak shifts towards higher $\kappa$ as $\sigma$ is increased. For example, for zero solidity factor, the peak power occurs at $\kappa=0.5$, while for $\sigma=0.01$, it occurs at $\kappa \cong 0.66$.

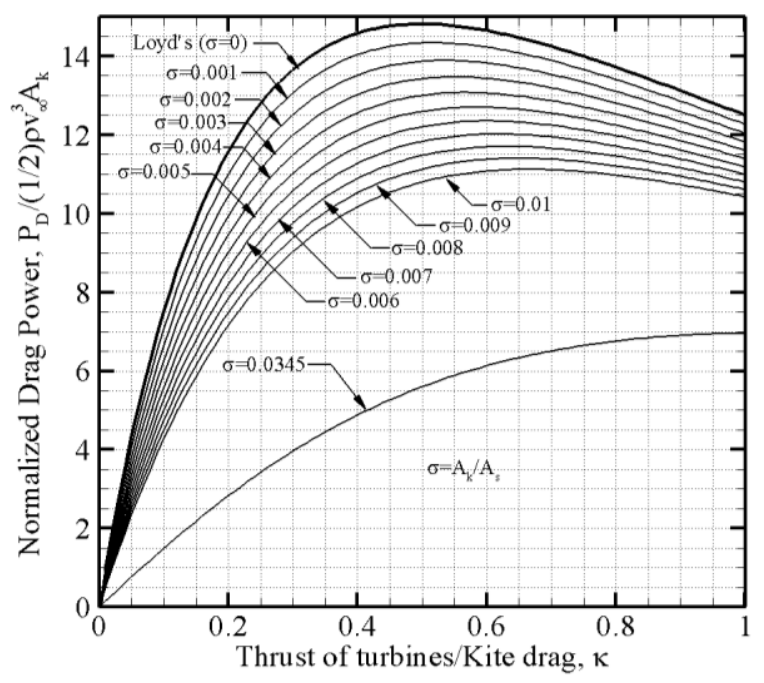

Figure 7. The normalized drag power as a function of the ratio of thrust of on-board turbines to kite drag, $\kappa$, for different values of the solidity factor, $\sigma$. For all the curves, $c_{L}=1.0$ and $\left(c_{L} / c_{D}\right)=10$.

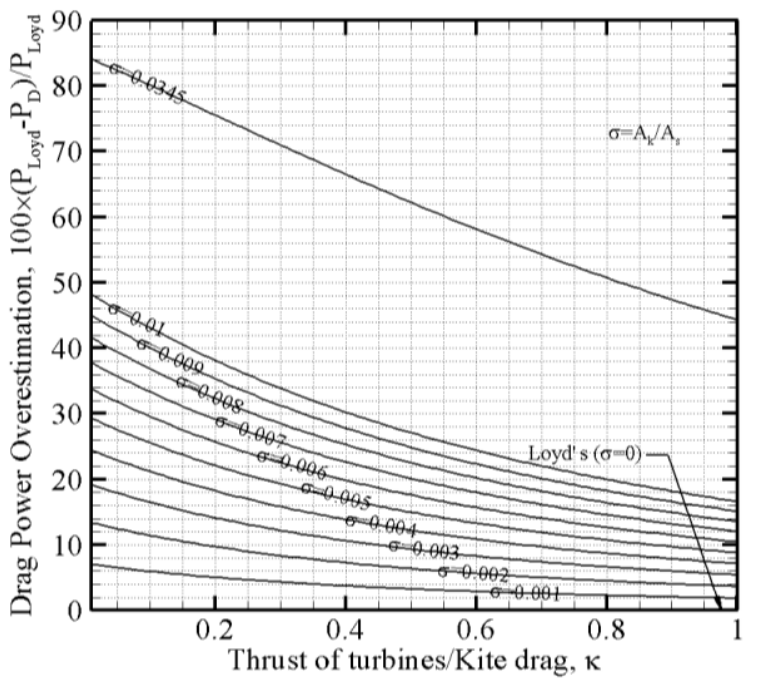

Figure 8. Drag power overestimation as a function of the ratio of thrust of on-board turbines to kite drag, $\kappa$, for different values of the solidity factor, $\sigma$. For all the curves, $c_{L}=1.0$ and $\left(c_{L} / c_{D}\right)=10$.

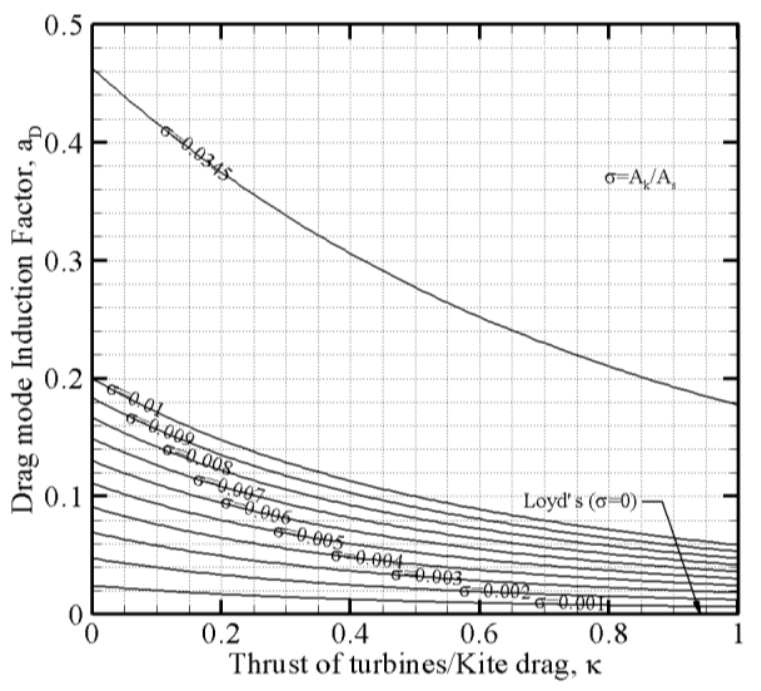

Figure 9. Induction factor in drag mode as a function of the ratio of thrust of on-board turbines to kite drag, $\kappa$, for different values of the solidity factor, $\sigma$. For all the curves, $c_{L}=1.0$ and $\left(c_{L} / c_{D}\right)=10$.

Fig. 8 shows how much overestimation is made in drag power calculation if one uses Loyd's formulation (i.e. neglecting the induction factor) instead of the formulation presented in this paper. As seen, the amount of overestimation is dependent on both the ratio of thrust of onboard turbines to kite drag and the solidity factor: it becomes larger when the solidity factor is increased and the thrust to drag ratio is decreased. For example, for $\sigma=0.001$, drag power overestimation varies between $2 \%($ at $\kappa=1$ ) and $7 \%$ (at $\kappa=0.01$ ), while for $\sigma=$ 0.01 , that is between $17 \%$ (at $\kappa=1$ ) and $48 \%$ (at $\kappa=0.01$ ).

Fig. 9 shows variation of the induction factor in drag mode, $a_{D}$, as a function of the ratio of thrust of on-board turbines to kite drag, $k$, for different values of the solidity factor, i.e. $\sigma=A_{k} / A_{s}=0.001$ to 0.01 . As expected from Eq. 28, the induction factor for a drag mode kite increases, and may become quite significant, as $\sigma$ increases and/or $k$ decreases. 
As seen from Figs. 4 and 7, the peak normalized power in lift mode for a kite with $\sigma=0$ (i.e. infinite capture area) is approximately 14.81 (assuming $c_{L}=1.0$ and $\left(c_{L} / c_{D}\right)=10$ ) which is the same in drag mode. This agrees with Loyd's results. However, for a non-zero solidity factor (thus a non-zero induction factor) system, the maximum drag power is greater than the maximum lift power. One may then quickly jump into conclusion that the drag mode power generation is more efficient than the lift mode power generation. However, this conclusion may be wrong as a kite in lift mode normally has a much smaller solidity factor (as it sweeps a large area during the power stroke) compared to a kite with the same rated power in drag mode. Furthermore, the average induction factor may be reduced when taking into account that no wind power is extracted during the retraction part of a pumping cycle.

\section{CONCLUDING REMARKS}

In this paper, the actuator disc theory was extended and applied to crosswind kite systems. In contrast to previous studies in which the effect of kite on slowing the incoming flow has been neglected, in the present paper the effect of axial induction factor was considered. Expressions for induction factor and harvestable power in both lift and drag modes were obtained. It was shown that for an induction factor of zero, the resulting expressions for power are equivalent to those obtained by (Loyd, 1980). Numerical results showed that the harvestable power is strongly dependent on the solidity factor of the kite. It was shown that the maximum lift power is independent of the ratio of reel-out speed to freestream velocity, whereas the maximum drag power varies with the ratio of on-board turbines thrust to kite drag. It was also shown that the induction factor for a crosswind kite system is not necessarily negligible, and that neglecting even a small induction factor in power calculation may result in a significant overestimation of power.

\section{ACKNOWLEDGMENTS}

This research was conducted at New Leaf Management. The first author is also grateful to the Faculty of Engineering and Computer Science of Concordia University for a start-up research grant.

\section{APPENDIX}

\section{Bernoulli's equation for flow in contact with a moving actuator disc}

Referring to Fig. A.1, the top figure shows a moving actuator disc at time $t$ with an "inlet" streamline extending between points 1 and 2 and an "outlet" streamline from point 3 to 4 . The bottom figure shows the same system at time $t+\Delta t$. Points 1 and 2 are located, respectively, sufficiently far upstream and downstream of the disc, where the (absolute) flow velocities are, respectively, $v_{\infty}$ and $v_{o}$. Points 2 and 3 are located just in front and behind the actuator disc and are moving with the disc at disc speed, $v_{d}$; the (absolute) flow velocity at both these points is denoted by $v$. It is assumed that the velocity of the flow changes linearly between points 1 and 2 and between points 3 and 4. ${ }^{7}$ Two different coordinate systems are used: a) fixed/inertial coordinate systems represented by $X_{i}, i=1,2$, and b) moving

${ }^{7}$ This is consistent with the general actuator disc theory; for more details, refer to (Sorensen, 2016). coordinate systems represented by $x_{i}, i=1,2$; the indices 1 and 2 refer, respectively, to the points on the left- and right-hand sides of the disc.

Applying unsteady Bernoulli's equation to the streamline between points 1 and 2, and between points 3 and 4 (refer to(White, 2008)) leads to:

$$
\begin{aligned}
& \int_{1}^{2} \rho\left(\frac{\partial v}{\partial t}\right) \mathrm{d} s+p_{d-}-p_{\infty}+\frac{1}{2} \rho\left(v^{2}-v_{\infty}^{2}\right)=0 \\
& \int_{3}^{4} \rho\left(\frac{\partial v}{\partial t}\right) \mathrm{d} s+p_{o}-p_{d+}+\frac{1}{2} \rho\left(v_{o}^{2}-v^{2}\right)=0
\end{aligned}
$$

where $p_{d_{-}}$and $p_{d_{+}}$are the static pressure at points 2 and 3 , respectively.

One may find the flow velocities at arbitrary points A and B, shown in Fig. A.1, as

$$
\begin{gathered}
v_{f 1}\left(x_{1}, t\right)=v_{\infty}+\left(\frac{v-v_{\infty}}{\ell_{1}}\right) x_{1}, \quad 0 \leq x_{1} \leq \ell_{1} \\
v_{f 2}\left(x_{2}, t\right)=v_{o}+\left(\frac{v_{o}-v}{\ell_{2}}\right) x_{2}, \quad-\ell_{2} \leq x_{1} \leq 0 .
\end{gathered}
$$

From Eqs. A.3 and A.4, one may find the time derivative of the flow velocities as

$$
\begin{aligned}
& \frac{\partial v_{f 1}}{\partial t}=\left(\frac{v-v_{\infty}}{\ell_{1}}\right) \frac{\partial x_{1}}{\partial t} \\
& \frac{\partial v_{f 2}}{\partial t}=\left(\frac{v_{o}-v}{\ell_{2}}\right) \frac{\partial x_{2}}{\partial t} .
\end{aligned}
$$

Using Fig. A.1, $\left(\partial x_{1} / \partial t\right)$ and $\left(\partial x_{2} / \partial t\right)$ may be obtained as follows

$$
\begin{aligned}
& \frac{\partial x_{1}}{\partial t}=\lim _{\Delta t \rightarrow 0} \frac{x_{1}(t+\Delta t)-x_{1}(t)}{\Delta t}=-\lim _{\Delta t \rightarrow 0} \frac{\Delta s}{\Delta t}=-\frac{\partial s}{\partial t}=-v_{d}, \\
& \frac{\partial x_{2}}{\partial t}=\lim _{\Delta t \rightarrow 0} \frac{x_{2}(t+\Delta t)-x_{2}(t)}{\Delta t}=-\lim _{\Delta t \rightarrow 0} \frac{\Delta s}{\Delta t}=-\frac{\partial s}{\partial t}=-v_{d} .
\end{aligned}
$$

Eqs. A.5 and A.6 may then be re-written as

$$
\begin{aligned}
& \frac{\partial v_{f 1}}{\partial t}=-v_{d}\left(\frac{v-v_{\infty}}{\ell_{1}}\right), \\
& \frac{\partial v_{f 2}}{\partial t}=-v_{d}\left(\frac{v_{o}-v}{\ell_{2}}\right) .
\end{aligned}
$$

Substituting Eqs. A.9 and A.10 into Eqs. A.1 and A.2, respectively, and performing integrations yields

$$
\begin{gathered}
-\rho v_{d}\left(v-v_{\infty}\right)+p_{d-}-p_{\infty}+\frac{1}{2} \rho\left(v^{2}-v_{\infty}^{2}\right)=0 \\
-\rho v_{d}\left(v_{o}-v\right)+p_{o}-p_{d+}+\frac{1}{2} \rho\left(v_{o}^{2}-v^{2}\right)=0
\end{gathered}
$$

which are further simplified to

$$
\begin{aligned}
& p_{\infty}+\frac{1}{2} \rho\left(v_{\infty}^{2}-v_{d}^{2}\right)=p_{d-}+\frac{1}{2} \rho v_{r}^{2}, \\
& p_{d+}+\frac{1}{2} \rho v_{r}^{2}=p_{o}+\frac{1}{2} \rho\left(v_{o}^{2}-v_{d}^{2}\right),
\end{aligned}
$$

where $v_{r}=v-v_{d}$ is the relative flow velocity at the actuator disc. 


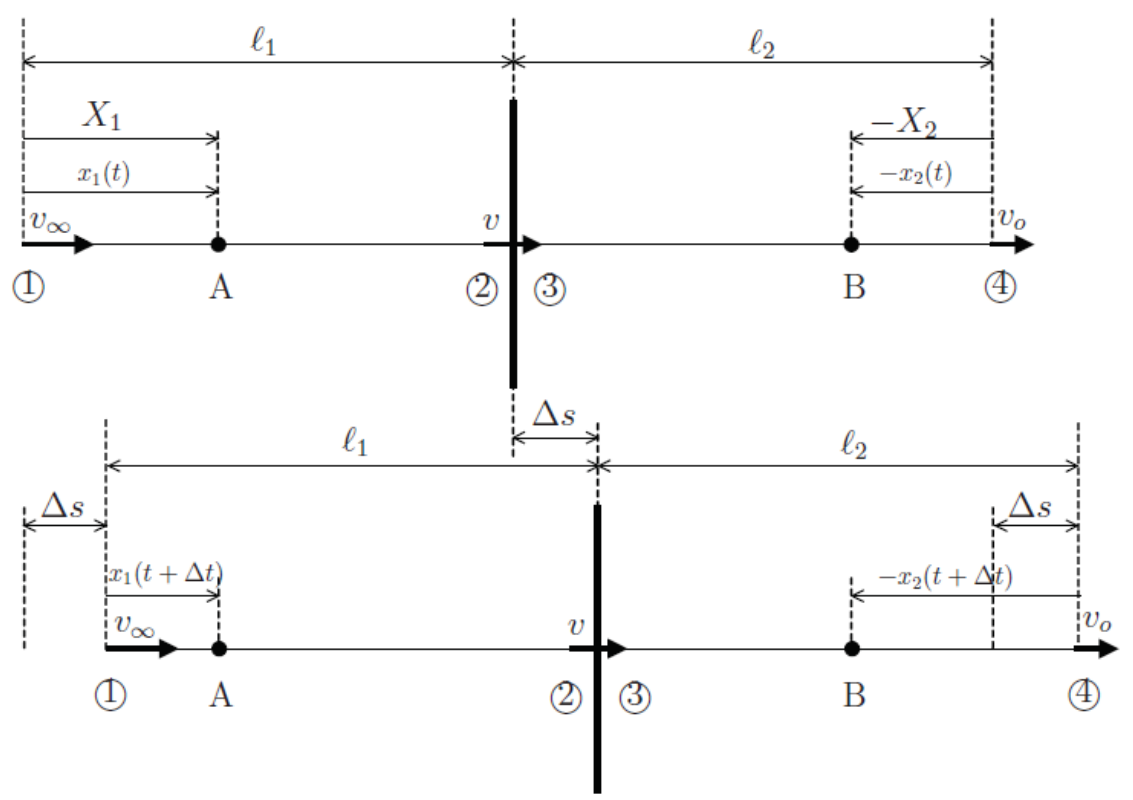

Figure A.1. A schematic showing the effect of a moving disc on the flow field. The top figure shows the situation at time $t$, while the bottom one corresponds to time $t+\Delta t$. Points A and B are fixed in space, while points 1 to 4 are moving with the disc; in fact, point 1 is where the flow velocity is $v_{\infty}$; point 4 is where the flow velocity is $v_{o}$, and points 2 and 3 are just before and after the disc; $X_{i}(i=1,2)$ are inertial coordinate systems, while $x_{i}(i=1,2)$ are moving with the disc. Also, $\ell_{1}$ and $\ell_{2}$ represent the extent of the streamtube, respectively, upstream and downstream of the disc; $\Delta s$ is the distance travelled during the period $\Delta t$; lastly, $v$ represents the absolute flow velocity at the disc.

\section{REFERENCES}

Ahrens, U., Diehl, M., \& Schmehl, R. (2013). Airborne Wind Energy. Berlin: Springer.

Bazilevs, Y., Hsu, M., Akkerman, I., Wright, S., Takizawa, K., Henicke, B., et al. (2011). 3D simulation of wind turbine rotors at full scale. Part I: Geometry modeling and aerodynamics. International Journal for Numerical Methods in Fluids, 65, 207-235.

Burton, T., Sharpe, D., Jenkins, N., \& Bossanyi, E. (2001). Wind Energy Handbook. West Sussex: John Wiley \& Sons, Ltd.

Cherubini, A. a. (2015). Airborne Wind Energy Systems: A review of the technologies. Renewable and Sustainable Energy Reviews, 51, 1461-1476.

Costello, S., Costello, C., François, G., \& Bonvin, D. (2015). Analysis of the maximum efficiency of kite-power systems. Journal of Renewable and Sustainable Energy, 7 (5), 053108.

Loyd, M. L. (1980). Crosswind Kite Power. Journal of Energy (AIAA), 4 (3), 106-111.

Okulov, V., \& van Kuik, G. A. (2009). The Betz-Joukowsky limit for the maximum power coefficient of wind turbines. International Scientific Journal for Alternative Energy and Ecology, 9, 106-111.

Sorensen, J. N. (2016). General Momentum Theory for Horizontal Axis Wind Turbines. Springer.

White, F. M. (2008). Fluid Mechanics (Sixth ed.). New York: McGraw-Hill.
Wilson, R. E., \& Lissaman, P. B. (1974). Applied aerodynamics of wind power machines. Corvallis (USA): Oregon State University. 